\title{
Effect of Treatment With Direct Acting Antivirals on Glucose and Lipid Metabolism in Egyptian Patients With Chronic Hepatitis C
}

\author{
Aalaa Mokhtar Ahmed ${ }^{(1)}$, Dina Ismail Attia ${ }^{(2)}$, Hossam Sabry Ghoniem ${ }^{(3)}$ and Yehia Sameh \\ Korriem $^{(4)}$ \\ (1) Tropical Medicine department, Faculty of Medicine, Beni-Suef University. \\ (2) Professor and head of Tropical Medicine department, Faculty of Medicine, Beni-Suef \\ University. \\ (3) Assistant professor at Tropical Medicine department, Faculty of Medicine, Beni-Suef \\ University. \\ (4) Lecturer at Tropical Medicine department, Faculty of Medicine, Beni-Suef University.
}

Corresponding author: Aalaa Mokhtar Ahmed

E-mail address: dr.hams91@gmail.com

Tel: 01012995217

\begin{abstract}
The aim of this study is to evaluate the effect of the different direct acting antivirals regimens available for treatment of Egyptian patients with chronic hepatitis post hepatitis C virus (HCV) infection as regards the changes in blood glucose and lipid metabolism.

This is prospective observational study conducted on 45 naïve patients with chronic hepatitis $\mathrm{C}$ infection. All the patients were subjected to routine; laboratory investigation, fasting serum insulin, lipid profile and abdominal ultrasound before receiving direct acting antivirals (DAAs) and at 12 weeks after end of treatment at sustained virologcal response (SVR) 12.

A significant increase of cholesterol, triglycerides and HDL at SVR 12 (P-value <0.001, 0.007 and $<0.001$ ) was observed.

significant decrease in both ALT \& AST p.value 0.001 for both the same for total bilirubin p.value
\end{abstract}

0.001, While serum albumin increased p.value 0.012 were also found at SVR 12 .

There was a significant decrease of insulin, fasting blood glucose FBG, 2 hours postprandial glucose and the HOMA IR 12 weeks after treatment at SVR 12 (P-value $=0.010,0.006$, $<0$;;001 and 0.002, respectively).

Fibrosis index 4 (FIB-4) showed significant improvement (P-value <0.001).

\section{Keywords}

Direct acting antiviral, glucose metabolism, lipid metabolism. 


\section{Introduction}

Chronic hepatitis $\mathrm{C}(\mathrm{HCV})$ virus infects an estimated 170 million individuals worldwide, with significant associated morbidity and mortality [1].

According to World Health Organization (WHO), Egypt has the highest prevalence of hepatitis $\mathrm{C}$ virus ( $\mathrm{HCV})$, where the results of blood screening and testing for the Egyptian blood donors showed $20 \%$ positive for $\mathrm{HCV}$. A recently published Egypt Health Issues Survey (EHIS) in 2015 on a nationally representative sample showed that $10 \%$ of Egyptians between 15 - 59 years of age had been infected with HCV infection, while $7 \%$ are chronic active hepatitis C patients [2].

The landscape of HCV management is changing dramatically with the advent and approval of the new oral direct acting antiviral (DAA) medications [3].

With so many resources being placed into the development of novel therapies, investigation into how $\mathrm{HCV}$ interacts with other metabolic processes remains of interest to the Hepatology community [4].

It is widely recognized that $\mathrm{HCV}$ infection is associated with several metabolic derangements including hypolipidemia, hepatic steatosis and metabolic syndrome [5], Several studies have also confirmed a multifold increase in the prevalence of glucose abnormalities in patients with HCV as compared to control [6].
Changed lipid levels (total cholesterol, triglyceride) and improved insulin resistance after treatment with interferon-based therapy are well known. But here is a shortage in data on changes in lipid levels and glycemic parameters with the newer DAAs [7].

\section{Patients and Methods}

Out of 250 patient attended to The Outpatient Clinic for HCV treatment of Tropical Medicine Department, Beni-suef University Hospitals between September 2018 and July 2019, 45 patients were enrolled to our prospective observational study as they met the inclusion criteria.

The inclusion criteria were age between 18-75 years old with positive HCV RNA results by quantitative PCR, naïve patients, egyptian patients, cirrhotic patients with Child Pugh score A \& B and patients accepting to sign the written informed consent.

Patients with the following conditions were excluded from this study; Child-Pugh C cirrhotic patients active alcohol intake, presence of ascites, jaundice or hepatic encephalopathy, presence of known other concomitant liver disease such as: chronic hepatitis B, hemochromatosis, Wilson's disease, autoimmune hepatitis and primary biliary cirrhosis, pregnancy or inability to use effective contraception, patients diagnosed with diabetes mellitus, presence of other organ failure especially end stage renal disease and heart failure and inability to provide written informed 
consent from the patient or one of his relatives in case of disability.

\subsection{Clinical and laboratory assessment:}

\section{All the patients were subjected to}

1-Complete history taking: with special emphasize on smoking history, alcohol intake, history and presence of diabetes mellitus. All the patients were thoroughly asked about the manifestations of liver cell failure (as jaundice, ascites, hepatic encephalopathy, hematemesis and melena), upper or lower gastrointestinal affection or other organ failure. Patients were also asked about any drug intake and chronic diseases as hypertension.

2-Clinical examination including: vital signs assessment, abdominal, chest and cardiac examination with special emphasis on manifestations of LCF (jaundice, bleeding tendency, ascites and lower limb edema).

Body mass index (BMI) and waist circumference were measured in all patients.

\section{3-Laboratory investigations including:}

serum samples will be taken after overnight (12 hours) fasting in order to assess;

-Full routine laboratory investigations: urine analysis, stool analysis and kidney function tests(serum creatinine, blood urea, serum sodium and serum potassium).

-Complete blood picture (CBC):Total leukocyte count with differential, Hemoglobin and platelet count.
-Liver function tests: Aspartate aminotransferase (AST), Alanine aminotransferase (ALT), serum albumin, bilirubin, prothrombin concentration and international normalized ratio( INR).

-Fasting blood glucose (FBG) \& 2 hours post prandial blood glucose (2h PP BG).

-Blood insulin level:

assessed by enzyme linked immunoassay (ELISA) using EDI human insulin ELISA kit.

-Insulin resistance (IR) was calculated for each patient by the homeostasis model for the assessment of IR (HOMA-IR) using the standard formula: HOMA-IR = fasting insulin $(\mathrm{uU} / \mathrm{mL}) \times$ fasting glucose $(\mathrm{mmol} / \mathrm{L}) / 22.5$.

-Lipid profile: total cholesterol (TC), highdensity lipoprotein cholesterol(HDL), low density lipoprotein cholesterol (LDL), and serum triglycerides(TGs).

\section{4-Abdominal Ultrasound:}

Real time abdominal ultrasound was performed after 6-8 hours fasting using a C3-5 array (Siemens acuson x 300, Enlargen,Germany) by an experienced sonographer. The procedure is computed for each patient with special emphasis on liver size, texture, liver surface, hepatic veins, and portal vein diameter, presence of splenomegaly or calcular gall bladder as well as presence or absence of ascites.

\section{5-AST to platelet ratio index(APRI):}

APRI is cheap and non-invasive tool to assess liver cirrhosis especially in conjunction with other testing like fibrosis index in our study. 


\section{6-Fibrosis index (FIB-4 Index):}

Calculated for all patients in order to assess stage of fibrosis in the least invasive procedure. FIB-4 was developed to correlate with Ishak levels of fibrosis (by biopsy).

\section{2 .Treatment protocol:}

All patients received DAAs for 12 weeks according to the guidelines of national committee for viral hepatitis treatment; 43 patients received dual therapy in the form of Sofosbuvir plus daclatasvir, only 2 patients received triple therapy in the form of Sofosbuvir+daclatasvir+ ribavirin.

\subsection{Follow up:}

All patients were asked about appearance of jaundice, ascites or other manifestations of liver cell failure, clinical side effects of DAAs All the collected data were tabulated and analyzed using SPSS v. 25 (Statistical Package for Social science) for Windows.

Description of variables was presented as follows; description of quantitative variables was presented in the form of mean, standard deviation (SD), median, minimum and maximum. Description of qualitative variables was presented in the form of numbers (No.) and

\section{Results:}

\subsection{Demographic distripution:}

We studied 45 naïve patients with $\mathrm{CHC}$, their demographic and metric data are detailed in Table 1. as headache, depression or manifestations of anemia (fatigue, shortness of breath, ...) were also asked for.

The following tests were repeated 24 weeks from starting treatment:

-HCV RNA quantitative PCR.

-Routine labs.

-Liver profile (ALT, AST, Albumin, bilirubin and INR).

•FBG \& 2 H PP BG.

-Fasting insulin level.

-HOMA -IR. And ipid profile (LDL), (HDL) ,(TC) and TGs

-FIB-4 index.

-APRI.

\subsection{Statistical methodology:}

percent's (\%). Paired T-test was used to detect the effect of treatment on the laboratory scale variables. The significance of the results was assessed in the form of P-value that was differentiated into non-significant when P-value $>0.05$, significant when $\mathrm{P}$-value $\leq 0.05$ and highly significant when $\mathrm{P}$-value $\leq 0.001$

Their mean age was $45.7 \pm 14$ years. $60 \%$ were females, none of them was smoker and only 2 patients were hypertensive.

The mean BMI was $24.9 \pm 4.9 \mathrm{~kg} / \mathrm{m} 2$ and ranged from 18 to $37 \mathrm{~kg} / \mathrm{m} 2$, and the mean waist circumference was $90.2 \pm 14.6$ and ranged from 65 to $112 \mathrm{~cm}$.

Table.1. Demographic and baseline characteristics of chronic hepatitis $\mathrm{C}$ patients: 


\begin{tabular}{|l|l|}
\hline Characteristics & $\begin{array}{l}\text { Values } \\
\text { N=45 no.(100\%) }\end{array}$ \\
\hline$\underline{\text { Age }}$ & $45.7 \pm 14$ \\
Mean \pm SD & $(20-70)$ \\
Range(min-max) & 45 \\
Median & $18(40)$ \\
\hline$\underline{\text { Sex }}$ & $27(60)$ \\
Males & $45(100)$ \\
\hline Females & Smoking \\
\hline No & data: \\
\hline
\end{tabular}

\section{2.biochemichal and metabolic data:}

In our study we evaluated the effect of DAAs with or without ribavirin on glucose metabolism in the form of fasting blood sugar, 2 hours post prandial blood sugar, serum insulin and insulin resistance and on lipid metabolism in the form of serum cholesterol, serum triglycerides, low density lipoproteins and high density lipoproteins, most of the baseline biochemical and metabolic vales of the 45 enrolled patients were in normal range (detailed in table 2).

Abdominal ultrasonography data for the 45 patienta are showed in table 3.

Table.2. baseline biochemical and metabolic data:

\begin{tabular}{|c|c|c|}
\hline $\begin{array}{l}\text { Parameters } \\
\text { treatment }\end{array}$ & Normal value & Mean \pm SD \\
\hline HB & $11.8-16 \mathrm{~g} \backslash \mathrm{dl}$ & $13.3 \pm 1.4$ \\
\hline TLC & $4-11 \times 10^{3} \mathrm{Imm}^{3}$ & $6.6 \pm 2.2$ \\
\hline PLT $\times 10^{3}$ & $150-450 \times 10^{3} \backslash \mathrm{mm}^{3}$ & $287.6 \pm 88.5$ \\
\hline T.BIL & $0.3-1.2 \mathrm{mg} \backslash \mathrm{dl}$ & $0.7 \pm 0.3$ \\
\hline ALT & $0-40$ U\L & $39.8 \pm 23.1$ \\
\hline AST & $0-40 \mathrm{U \backslash L}$ & $39.6 \pm 17.8$ \\
\hline PC & 100 & $89.9 \pm 11.4$ \\
\hline INR & 1 & $1.1 \pm 0.1$ \\
\hline ALB & $3.5-5.2 \mathrm{~g} \backslash \mathrm{dl}$ & $4.1 \pm 0.5$ \\
\hline Creatinine & $0.4-1.3 \mathrm{mgldl}$ & $0.8 \pm 0.3$ \\
\hline Cholesterol & $150-200 \mathrm{mg} \backslash \mathrm{dl}$ & $149.1 \pm 19.8$ \\
\hline LDL & $0-190 \mathrm{mg} \backslash \mathrm{dl}$ & $140.9 \pm 150.9$ \\
\hline HDL & $15-45 \mathrm{mg} \backslash \mathrm{dl}$ & $37.8 \pm 9.79$ \\
\hline TGs & $40-160 \mathrm{mgldl}$ & $121.2 \pm 18.4$ \\
\hline FBS & $70-105 \mathrm{mg} \backslash \mathrm{dl}$ & $94.9 \pm 16.8$ \\
\hline 2.H PP & Less than 140 & $120.9 \pm 12.7$ \\
\hline Insulin & $0-25$ mIU\l & $7.2 \pm 7.8$ \\
\hline HOMA.IR & Less than 2 & $1.6 \pm 1.9$ \\
\hline
\end{tabular}


Table.3. Baseline Abdominal ultrasound findings:

\begin{tabular}{|l|l|l|}
\hline U.S & Number(45) & Percent (100\%) \\
\hline Homogenous liver & 24 & 53.3 \\
\hline Bright liver & 13 & 28.9 \\
\hline Bright hepatomegaly & 1 & 2.2 \\
\hline Coarse liver & 6 & 13.3 \\
\hline Splenomegaly & 1 & 2.2 \\
\hline
\end{tabular}

Regarding glucose metabolism, there was a significant decrease of insulin, FBG, 2 hours postprandial glucose and the HOMA IR after treatment $(\mathrm{P}$-value $=0.010,0.006,<0.001$ and 0.002, respectively) (Table 5).

Regardin changes in liver function tests and liver enzymes, we found non-significant effect on platelets with P.value 0.405 , With significant decrease in both ALT \& AST p.value 0.001 for both the same for total bilirubin p.value 0.001, While serum albumin increased p.value 0.012 (Table.6)..

\subsection{Virological response:}

Among the enrolled patients 43 patients received sofospuvir plus daclatasvir $(\mathrm{SOF}+\mathrm{DCV})$ for 12 months and 2 patients received ribavirin $(\mathrm{SOF}+\mathrm{DCV}+\mathrm{RBV})$ in addition to them while all the 45 patients achieved sustained virological reponse after 24 weeks.

\subsection{Changes inbiochemical and metabolic data after SVR 12:}

Regarding the changes in lipid profile after DAAs therapy, we found a significant increase in cholesterol level p.value <0.001, a significant increase in triglycerides (TGs) p.value 0.007, and a significant increase in HDLp.value <0.001 at SVR12. However, we found a non-significant change in LDL p.value 0.678 (table 4). 
Table.4. changes in lipid profile:

\begin{tabular}{|l|l|l|l|l|}
\hline \multicolumn{2}{|l|}{ Lipid profile } & Mean & $\begin{array}{l}\text { Std. } \\
\text { Deviation }\end{array}$ & \\
\hline \multirow{2}{*}{ Cholesterol } & Pre treatment & 149 & 19.8 & \multirow{2}{*}{$<\mathbf{0 . 0 0 1 * *}$} \\
\cline { 2 - 4 } & Post treatment & $\mathbf{1 6 9 . 7}$ & 25.9 & \\
\hline \multirow{2}{*}{ TG } & Pre treatment & 121.2 & 18.4 & \multirow{2}{*}{$\mathbf{. 0 0 7}^{*}$} \\
\cline { 2 - 4 } & Post treatment & $\mathbf{1 3 1 . 7}$ & 21.3 & \\
\hline \multirow{2}{*}{ LDL } & Pre treatment & 140.9 & 15 & \multirow{2}{*}{0.678} \\
\cline { 2 - 4 } & Post treatment & 131.5 & 19.3 & \\
\hline \multirow{2}{*}{ HDL } & Pre treatment & 37.7 & 9.7 & \multirow{2}{*0.001}{$1^{* *}$} \\
\cline { 2 - 4 } & Post treatment & $\mathbf{4 3 . 7}$ & 11 & \\
\hline
\end{tabular}

Table.5. changes in blood sugar and insulin resistance:

\begin{tabular}{|c|c|c|c|c|}
\hline \multicolumn{2}{|c|}{ Parameters } & \multirow{2}{*}{$\begin{array}{l}\text { Mean } \\
7.17 \\
\end{array}$} & \multirow{2}{*}{$\begin{array}{l}\text { Std. } \\
\text { Deviation } \\
7.8\end{array}$} & \multirow{2}{*}{$\begin{array}{l}\text { P-value } \\
0.010^{*}\end{array}$} \\
\hline Insulin & Pre treatment & & & \\
\hline & Post treatment & 4.5 & 4.7 & \\
\hline \multirow[t]{2}{*}{ FBG } & Pre treatment & 94.9 & 16.8 & \multirow[t]{2}{*}{ 0.006* } \\
\hline & Post treatment & 87.1 & 13.9 & \\
\hline \multirow[t]{2}{*}{$2 \mathrm{hrPP}$} & Pre treatment & 120.9 & 12.7 & \multirow[t]{2}{*}{$<0.001^{* * *}$} \\
\hline & Post treatment & 112.5 & 12.5 & \\
\hline \multirow{2}{*}{$\begin{array}{l}\text { HOMA- } \\
\text { IR }\end{array}$} & Pre-treatment $($ median $=0.9)$ & 1.6 & 1.8 & \multirow[t]{2}{*}{$0.002 *$} \\
\hline & Post treatment $($ median $=0.5)$ & 0.9 & 0.8 & \\
\hline
\end{tabular}

Table.6. changes in liver finction tests and liver enzymes:

\begin{tabular}{|c|c|c|c|c|}
\hline \multicolumn{2}{|l|}{ Labs } & \multirow{2}{*}{$\begin{array}{l}\text { Mean } \\
287.6\end{array}$} & \multirow{2}{*}{$\begin{array}{l}\text { Std. } \\
\text { Deviation } \\
88.5\end{array}$} & \multirow{2}{*}{$\begin{array}{l}\text { P-value } \\
0.405\end{array}$} \\
\hline \multirow{2}{*}{ PLT } & Pre treatment & & & \\
\hline & Post treatment & 298 & 78.3 & \\
\hline \multirow[t]{2}{*}{ ALT } & Pre treatment & 39.8 & 23 & \multirow[t]{2}{*}{$<0.001 * *$} \\
\hline & Post treatment & 29.4 & 12.6 & \\
\hline \multirow[t]{2}{*}{ AST } & Pre treatment & 39.6 & 17.8 & \multirow[t]{2}{*}{$<0.001 * *$} \\
\hline & Post treatment & 27.8 & 11.4 & \\
\hline \multirow{2}{*}{$\begin{array}{l}\text { T.Bil } \\
\text {. }\end{array}$} & Pre treatment & 0.67 & 0.27 & \multirow[t]{2}{*}{$<0.001 * *$} \\
\hline & Post treatment & 0.56 & 0.22 & \\
\hline \multirow[t]{2}{*}{ ALB } & Pre treatment & 4.1 & 0.54 & \multirow[t]{2}{*}{$0.012^{*}$} \\
\hline & Post treatment & 4.2 & 0.46 & \\
\hline
\end{tabular}


We trien to find significant association between this improvement and different demographic and clinical factors but there was not any correlation.

predominance (socially not accepted to be smokers).only two of the patients had history of contact with canal water.

Diabetes mellitus was among the exclusion criteria and none of the patients had hypertension, while in El Sagheer 's study 9.2\% were hypertensive[8].

In our study patients' body mass index(BMI) was $24.9 \pm 4.9$ and ranged from 18 to 37 , most of patients were within normal BMI, in contrary to Doyle 's study where BMI was $30.0 \mathrm{~kg} / \mathrm{m} 2$ (SD 4.6) [ 9].

Our study reached $100 \%$ SVR 12 in patients who received ribavirin in combination with sofosbuvir and daclatasvir (not easy to treat group) or did not (easy to treat group) while in El Sagheer's study 99\% reached SVR12 where patients received sofosbuvir and semiprevir( only one patient did not reach SVR12) [8].

Meissner conducted a study in 2015 where patients were treated with SOF and RBV, whereas 17 of $55(31 \%)$ relapsed [10].

Regarding the changes in lipid profile after DAAs therapy, we found we found a significant increase in cholesterol level at SVR12 the same as recently reported by Jain et, al 2019 and EL Sagheer et, al. 2018 .

\subsection{Changes in FIB-4:}

As we studiyin the changes of FIB-4 before treatment and after $\mathrm{HCV}$ eradication, we found a significant improvement p.value $<0.001$.

\section{Discussion}

Changed lipid levels (total cholesterol, triglyceride) and improved insulin resistance after treatment with interferon-based therapy are well known. But here is a shortage in data on changes in lipid levels and glycemic parameters with the newer DAAs [7].

In our study we evaluated the effect of DAAs with or without ribavirin on glucose metabolism in the form of fasting blood sugar, 2 hours post prandial blood sugar, serum insulin and insulin resistance and on lipid metabolism in the form of serum cholesterol, serum triglycerides, low density lipoproteins and high density lipoproteins, 45 naïve patients were enrolled.

The mean age was $45.7 \pm 14$ years. Most of patients were females (60\%) while in the study conducted by Doyle and colleagues in 2019 the mean age was 54 years (standard deviation( SD) 11.6) and was $47.5 \pm 12.3$ years in the study performed by EI Sagheer in 2018 where 48 patients were males (60\%) [8] [9].

None of the enrolled patients were alcoholic or smoker most probably due to female 
This is compatible with the results in the study by Russo and colleagues in 2019 where a significant number of patients showed an improvement of HOMA-IR. However, this study showed insulin resistance in $60 \%$ of enrolled patients from the start which is not the caase in our study, this may be due to the largest number of patients enrolled in their research against the relatively small number in ours [12]. similarly EL Sagheer found significant improvement in insulin resistance in patients received SOF- SIM regimen [8].

On the contrary Doyle and her colleagues in 2019 observed no consistent on-treatment or post-SVR change in measures of glucose homeostasis, also HOMA-IR did not improve from baseline during the treatment phase or post-DAA dosing in the overall study population or any subgroup [9].

Messier's study in 2015 observed no difference in HOMA-IR between baseline, on-treatment and the post-treatment phase were observed in patients treated with an IFN-free regimen.

In our study we observed significant decrease in both fasting and 2 hours postprandial glucose levels in blood at SVR 12 and this means improvement in glucose homeostasis mechanisms and glycemic control in patients cured from $\mathrm{HCV}$ infection, this was also observed by Jain A et al 2019 where hemoglobin A1c improved in diabetic patients
On the other hand Inoue and colleagues found non-significant change in cholesterol level in patients who received sofosbuvir combined with ribavirin[11].

We also found a significant increase in triglycerides (TGs) which is similar to what was reported by Doyle and her colleagues in 2109, but unlike to what was found by EI Sagheer in2018 as they found a significant decrease in TGs after treatment [8] [9].

Similarly we found a significant increase in HDL at SVR12 which was similar to values found by El Sagheer 'study but unlikely to the non-significant change found by Russo and colleagues [8] [12].

we found a non-significant change in LDL Unlikely to Most of studies that configured the effect of $\mathrm{HCV}$ eradication on lipid profile in $\mathrm{CHC}$ where a significant increase in LDL after end of treatment and at SVR was observed as the in the study by Inoue T, et al 2019, doyle $M$ et al 2019 and Jain A et al 2019.

$\mathrm{HCV}$ is closely related to insulin resistance and it is thought that eradication of $\mathrm{HCV}$ infection would improve insulin resistance and glycemic control [13],

In our study most of the patients had normal baseline HOMA IR as insulin resistance diagnosed when HOMA IR >2 which challenges the previous statement, though, HOMA IR was significantly decreased at SVR 12. 
resistance but we couldn't establish any significant correlation.

\section{References:}

1. Bose $S$ and Ray R (2014): Hepatitis $C$ virus infection and insulin resistance. World $J$ Diabetes.;5(1):52-58

2. El-Zanaty F and Way A (2009): Egypt Demographic Health Survey 2008 (EDHS 2008). Cairo: Ministry of Health and Population, National Population Council.

3. Fried M, Buti M, Dore G, et al (2013): Oncedaily simeprevir (TMC435) with pegylated interferon and ribavirin in treatment-naive genotype 1 hepatitis $\mathrm{C}$ : the randomized PILLAR study. Hepatology; 58: 1918-29.

4. Zeuzem S, Berg T, Gane E, et al. (2014): Simeprevir increases rate of sustained virologic response among treatment experienced patients with HCV genotype-1 infection: a phase IIb trial. Gastroenterology; 146: 430-41.

5. Greenberg P, Rosman A, Eldeiry L, et al (2006): Decline in haemoglobin A1c values in diabetic patients receiving interferon-alpha and ribavirin for chronic hepatitis C. $J$ ViralHepatitis; 13: 613-617.

6. Lin et al.(2014): Hepatitis $C$ virus-associated cancer. Annu. Rev. Pathol., 10 (2014), pp. 345370

7. Jain A, Kalra B, Srivastava S, et al (2019): Effect of sofosbuvir and daclatasvir on lipid profile, glycemic control and quality of life enrolled in their study with a significant decrease in median levels of glycated hemoglobin (HbA1c) was observed [7].

We observed a significant improvement in liver enzymes and almost all liver function tests in enrolled patients at SVR 12 with.

Russo FP and his colleagues observed similar results regarding ALT, AST and ALB but with insignificant change in T.BIL through of the treatment journey of patients with interferon free regimens.

In order to assess hepatic fibrosis noninvasively we calculated fibrosis index 4 (FIB 4) where $<\mathbf{1 . 4 5}=>0-1$ fibrosis stage, $\mathbf{1 . 4 5}$ 3.25 $=>2-3$ and >3.25 $=>$ 4-6 fibrosis stage, we also calculated AST to platelet ratio index (APRI) where Significant fibrosis: APRI

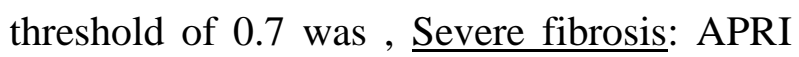
threshold of $1.0 \&$ Cirrhosis: APRI threshold of 1.0

Both FIB4 and APRI scores were normal at baseline for almost all enrolled patients but also showed significant improvement at SVR 12 unsimilar to what observed by Inoue in 2018 where there was not significant change in FIB 4 index and to what was found by Drazilova in 2018 with insignificant change in APRI score[14].

We studied this significant improvement in FIB-4 index and if it is associated to the age, gender, ultrasonographic features of liver and the change in TGs, cholesterol and insulin 
regimens of interferon-free direct-acting antivirals for patients infected with hepatitis $\mathrm{C}$ virus. Hepatology Research 48:203-212.

12. Russo FP, Zanetto A, Gambato $M$, et al (2019): Hepatitis $C$ virus eradication with direct acting antiviral improves insulin resistance. J Viral Hepat;2019:1-7.

13. Brandman D, Bacchetti P, Ayala $\mathbf{C}$, et al. (2012): Impact of insulin resistance on $\mathrm{HCV}$ treatment response and impact of $\mathrm{HCV}$ treatment on insulin sensitivity using direct measurements of insulin action. Diabetes Care, 35, 1090-1094.

14. Drazilova S, Janicko M, Skladany L, et al (2018): Glucose Metabolism Changes in Patients with Chronic Hepatitis C Treated with Direct Acting Antivirals. Can J Gastroenterol Hepatol.;2018: 6095097. index in chronic hepatitis $\mathrm{C}$, genotype 3 patients. Indian Journal of Gastroenterology; 38(1), 39-43.

8. El Sagheer G, Soliman E, Ahmad A, et al (2018): Study of changes in lipid profile and insulin resistance in Egyptian patients with chronic hepatitis $\mathrm{C}$ genotype 4 in the era of DAAs. Libyan J Med.;13(1) : 1435124.

9. Doyle M, Galanakis C, Mulvihill E, et al (2019): Hepatitis C Direct Acting Antivirals and Ribavirin Modify Lipid but not Glucose Parameters. Cells; 8: 4-17.

10. Meissner E, Lee Y, Osinusi A, et al (2015): Effect of sofosbuvir and ribavirin treatment on peripheral and hepatic lipid metabolism in chronic hepatitis $\mathrm{C}$ virus, genotype 1 -infected patients. Hepatology.;61(3):790-801.

11. Inoue T, Goto T, Iio E, et al (2018): Changes in serum lipid profiles caused by three 